\title{
Modification of Potato Starch by Acetylmalic Acid Chloroanhydride and Physicochemical Research of the New Product
}

\author{
Oksana Shulga $\left(D,{ }^{1}\right.$ Natalya Simurova, ${ }^{2}$ Sergii Shulga, ${ }^{2}$ and Jelyzaveta Smirnova ${ }^{3}$ \\ ${ }^{1}$ Department of Foodstuff Expertise, National University of Food Technologies, Kyiv, Ukraine \\ ${ }^{2}$ Department of Food Chemistry, National University of Food Technologies, Kyiv, Ukraine \\ ${ }^{3}$ Department of Foreign Languages Professional Direction, National University of Food Technologies, Kyiv, Ukraine
}

Correspondence should be addressed to Oksana Shulga; shulgaos@nuft.edu.ua

Received 24 October 2017; Accepted 29 November 2017; Published 16 January 2018

Academic Editor: Yeong-Soon Gal

Copyright (C) 2018 Oksana Shulga et al. This is an open access article distributed under the Creative Commons Attribution License, which permits unrestricted use, distribution, and reproduction in any medium, provided the original work is properly cited.

The article presents the research results of the product's properties of potato starch modification by acetylmalic acid chloroanhydride. Modification of potato starch has been carried out and has been confirmed by elemental analysis. In the infrared spectra, changes in the frequency oscillations of native starch in the noncharacteristic region have occurred: the frequency of oscillations at $981.81 \mathrm{~cm}^{-1}$ has increased and in the spectrum of modified starch has been at $1024.82 \mathrm{~cm}^{-1}$; the band with frequency of oscillations of $923.07 \mathrm{~cm}^{-1}$ has shifted to $866.66 \mathrm{~cm}^{-1}$, and the band with frequency of oscillations of $609.79 \mathrm{~cm}^{-1}$ has shifted to $672.22 \mathrm{~cm}^{-1}$, indicating the change in noncharacteristic region of the native starch sample after acylation. The properties of obtained modified product have been studied and this modification has appeared to change the shape of moisture and starch bonds, along with decreasing appearance of grains and reduced degree of crystallinity from 12 to $4 \%$.

\section{Introduction}

Modified starch is used in various sectors of food industry: confectionery, baking, brewing, meat and dairy, and foodconcentrate, for sale through the trading network, in the catering system, and for technical purposes (in the textile, in paper industry, and for drilling wells). Modified starches refer to nutritional supplements with the indices E1400-E1451. Among chemically modified starches, acetylated [1] oxyalkylated, phosphate and succinate are known. Chloric, phosphoric, and sulphuric acids; potassium hydroxide; ammonium persulphate; phosphorus chloroxide; acetic anhydride or acetic acid; vinyl acetate; derivatives of amber acid are considered to be modifiers.

Starch has been recently used more often for production of biodegradable packaging materials. However, there are some restrictions on its use in this field: low moisture resistance and low mechanical properties. Modification of starch changes the structure of starch, as a result of which its properties change, which allows expanding the possibilities of practical application. That is why work in the direction of modification of starch for demands in various sectors of economy is needed and is still going on. Thus, the authors [2] have made cassava starch modification by propionic acid anhydride to improve interfacial adhesion of polyurethane. The authors [3] propose corn and amaranth starch modification from octane succinyl derivatives in order to improve the emulsification properties and to use in encapsulation. Scientists [4] have made chemical modification of sago starch by reacting esterification with fatty acid chlorides in the absence of organic solvent to produce nonpolar sago ether.

Corn starch esters synthesis with fatty acids has been carried out using vinyl laurate and stearate as reagents [5].

Some authors propose a method for synthesizing starch esters by fatty acids without organic solvents as the most effective [6].

Scientists [7] propose starch esterification by octanol, lauryl, and palmitoyl chloride.

In order to obtain hydrophobically modified starch, esterification of starch was carried out by chlorides with longchain fatty acids $\left(\mathrm{C}_{8}, \mathrm{C}_{12}\right.$, and $\left.\mathrm{C}_{16}\right)$ [8]. 
The properties of corn starch fatty acids higher esters have been studied by the authors [9].

The authors [10] modified potato starch by butyryl chlorides, capryloyl, lauryl, palmitoyl, and oleyl.

Thus, this work in the field of starch modification is considered to be relevant and is going on in order to obtain starch with set technological properties. The problem of improving modification method, which should be noncomplicated and cost-effective, is also relevant.

\section{Materials and Methods}

2.1. Materials. Potato starch produced by LLC "August-Kyi" and malic acid (E 296) were used along with the following solvents: DMSO, DMF, methanol, ethanol.
Starch was prepared as follows: $100.02 \mathrm{~g}$ of potato starch was placed into a $500 \mathrm{~cm}^{3}$ flask, connected to a water jet pump and heated on a boiling water bath for 8 hours. Product yield was $88.00 \mathrm{~g}$ (loss due to drying is $12 \%$ ).

2.2. Purification of DMSO. $300 \mathrm{~cm}^{3}$ of DMSO was maintained over $\mathrm{CaO}$ at the temperature of $400^{\circ} \mathrm{C}$ for 2 days. Then DMSO was moved to a flask of $500 \mathrm{~cm}^{3}$ adding $30 \mathrm{~cm}^{3}$ of dry benzene. Water remains were discharged at atmospheric pressure in the form of azeotrope with benzene; then DMSO was distilled in vacuum of a water jet pump.

\subsection{Acidification of Malic Acid by Acetic Anhydride}<smiles>CC(=O)OCC(CC(=O)O)OC(C)=O</smiles>

Into a $100 \mathrm{~cm}^{3}$ reactor equipped with a magnetic stirrer, a heater, a thermometer, and a refrigerator with a gas meter, $26.8 \mathrm{~g}(0.2 \mathrm{M})$ of malic acid was added, adding $24.5 \mathrm{~g}(0.24 \mathrm{M})$ of acetic anhydride and 1 drop of sulphuric acid, while stirring the mixture stood during 1.5 hours at the temperature of $50-60^{\circ} \mathrm{C}$. Then the temperature was raised to $90^{\circ} \mathrm{C}$ and the mixture was stirred for 0.5 hours. Acetic anhydride and acetic acid excess was discharged into water jet pump vacuum.

$28 \mathrm{~g}$ of product was obtained, which was used without further purification.

\subsection{Synthesis of Acetylmalic Acid Chloroanhydride}<smiles>CC(=O)OC(CC(=O)O)C(=O)Cl</smiles>

$28.0 \mathrm{~g}(0.13 \mathrm{M})$ of acetylmalic acid was placed into a $100 \mathrm{~cm}^{3}$ reactor equipped with a magnetic stirrer with a heater, a dropping funnel, a thermometer, and a reflux condenser with a gas meter and a gas transfer tube. When stirring through a dropping funnel, $34.0 \mathrm{~g}(0.286 \mathrm{M})$ of $\mathrm{SOCl}_{2}$ was added in small portions. We observed calm gas release and slight heating of reaction mass. In $1 \mathrm{~h}$ a drop of DMF was added, with the temperature being maintained at $50-60^{\circ} \mathrm{C}$ for the next 5 hours. $\mathrm{SOCl}_{2}$ excess remained in water jet pump vacuum; $32 \mathrm{~g}$ of product has been obtained which was used without further purification.

2.5. Esterification of Starch by Acetylmalic Acid Chloride. $9 \mathrm{~g}\left(0.05 \mathrm{M}\right.$ per glucose) of dried starch and $300 \mathrm{~cm}^{3}$ of dried DMSO were placed in a three-speed $500 \mathrm{~cm}^{3}$ reactor equipped with a magnetic stirrer with a heater, a dropping funnel, a thermometer, and a reflux condenser with a gas meter and a gas transfer tube. The mixture was stirred for $3 \mathrm{~h}$ at the temperature of $70^{\circ} \mathrm{C}$ to form a clear, colorless jelly. Then they stopped heating and added $0.5 \mathrm{~g}$ of $\mathrm{K}_{2} \mathrm{CO}_{3}$ (about $2 \%$ of the starch weight). When stirred, $32 \mathrm{~g}(0.15 \mathrm{M})$ of acetylmalic acid chlorohydride was added dropwise. Reaction mass became thicker and, in 1 hour, it turned into a very dense, light yellow color. Then mixture was heated again while being stirred for further $2 \mathrm{~h}$ at the temperature of $50-60^{\circ} \mathrm{C}$. Reaction mixture was left at the room temperature overnight. Then, $200 \mathrm{~cm}^{3}$ of methanol was added and vigorously stirred for 1 hour. A dropped white precipitate was filtered off in water jet pump vacuum and washed with distilled water $\left(2 \times 50 \mathrm{~cm}^{3}\right)$, ethanol $\left(2 \times 50 \mathrm{~cm}^{3}\right)$. The resulting product was dried in the air at the room temperature.

\section{Research Methods}

An infrared test was performed by the Nexus-475, Nicolet, a $\mathrm{KBr}$ tablet. 


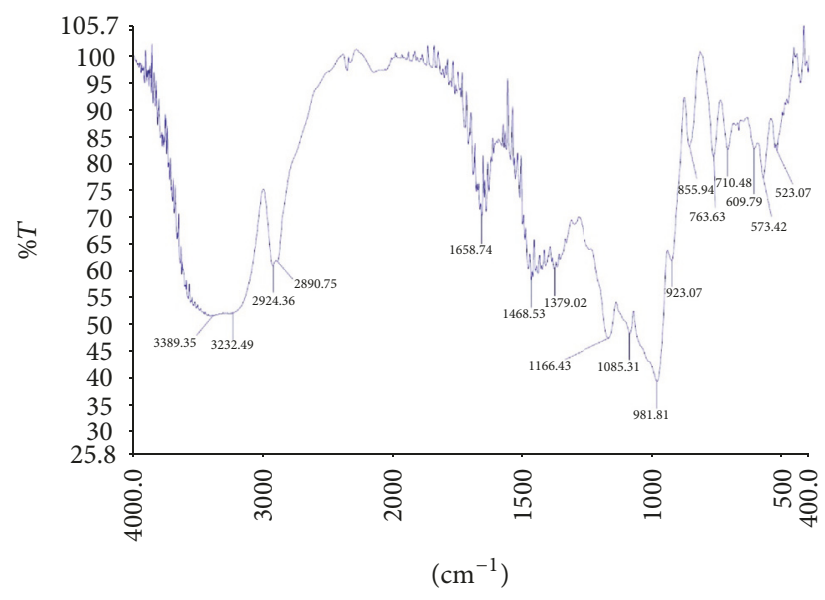

(a)

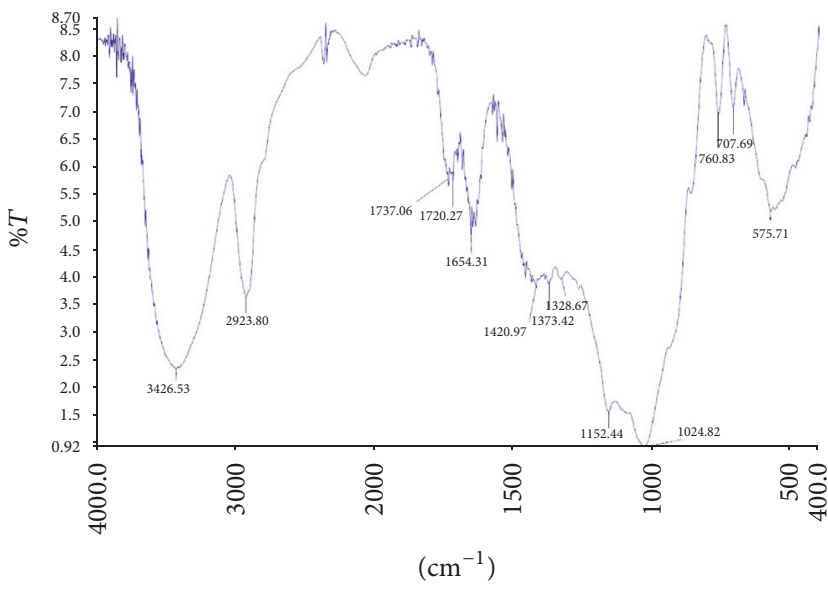

(b)

FIGURE 1: IR spectra of native (a) and modified (b) potato starch.

$\mathrm{X}$-ray diffraction analysis was carried out by the DRON$3 \mathrm{M}$ device in $\mathrm{CuK} \alpha$ emission with Ni filter; $U=35 \mathrm{kV}$, $I=20 \mathrm{~mA}$; counterdisplacement angle $\Delta 2 \Theta$ is $0,04^{\circ}$; time of intensity reckoning is $3 \mathrm{~s}$.

Thermogravimetric research was carried out by the Q1500B device.

NMR spectra were recorded by the Mercury NMR spectrometer, Varian, $400 \mathrm{MHz}$ in DMSO- $\mathrm{d}_{6}$.

\section{Results and Discussion}

4.1. IR Research. Changes resulting in acylation reaction of potato starch by malic acid chloride are confirmed by a number of factors. Thus, in infrared spectrum of modified starch (Figure 1(b)), maximum at $1737.06 \mathrm{~cm}^{-1}$ has appeared, which is typical for $\mathrm{C}=\mathrm{O}$ in the ester group, which has appeared in modified starch as a result of acylation reaction.

The nature of the band of valence vibrations $(\nu \mathrm{ON})$ in the IR spectra of potato starch (Figure 1(a)) and modified starch (Figure 1(b)) is a reliable confirmation of positive result of acylation reaction of starch by malic acid chloride. In the spectrum of native starch sample there is a sufficiently wide, intense band at $3389.35 \mathrm{~cm}^{-1}$, and, in the spectrum of modified sample, the band is more intense and less broad and lies in the region of stronger vibrations at $3426.53 \mathrm{~cm}^{-1}$. It is known [11] that the position and nature of $\nu \mathrm{ON}$ band depend on the degree of participation of hydroxyl group in hydrogen bonds. Hydrogen bonds reduce the frequency of oscillations. The hydroxyl group that participates in intermolecular hydrogen bonding is characterized by wide intensive absorption maximum of $3200-3600 \mathrm{~cm}^{-1}$.

Consequently, in IR spectrum of native starch, the band of valence vibrations $\nu \mathrm{ON}$ is wider and less intense than that of modified starch and lies at $3389.35 \mathrm{~cm}^{-1}$, and, in modified starch spectrum, this band is narrower and more intense (see Figure 1) and lies at $3426.53 \mathrm{~cm}^{-1}$. This confirms the fact that in modified starch there are fewer hydrogen bonds because less free hydroxyl groups are the part of their esterification, which also has affected the nature and frequency of oscillations of $\nu \mathrm{ON}$ acetylated potato starch.

In addition, when comparing infrared spectra of native and modified starches (Figure 1), it has been found that spectra under investigation have different oscillations in a noncharacteristic region. It is known [12] that numerous valent oscillations of the C-C, C-N, N-O and deformation oscillations are manifested in this area. This area of oscillation of a molecule carbon skeleton sharply reacts to minor changes in molecule structure.

Noncharacteristic oscillations form the main part of the spectrum and for each substance constitute their own unique set of bands [11, 13] and it is this factor that is used to determine identity of substances under study.

In IR spectrum of native starch (Figure 1(a)), there are a number of oscillations in noncharacteristic region, in particular $981.1 \mathrm{~cm}^{-1}, 923.07 \mathrm{~cm}^{-1}, 855.94 \mathrm{~cm}^{-1}, 763.63 \mathrm{~cm}^{-1}$, $710.48 \mathrm{~cm}^{-1}, 609.79 \mathrm{~cm}^{-1}, 573.12 \mathrm{~cm}^{-1}$, and $523.12 \mathrm{~cm}^{-1}$, and in spectrum of modified starch (Figure 1(b)) $-1024.82 \mathrm{~cm}^{-1}$, $866.66 \mathrm{~cm}^{-1}, 760.83 \mathrm{~cm}^{-1}, 707.69 \mathrm{~cm}^{-1}, 672.22 \mathrm{~cm}^{-1}$, and $575.71 \mathrm{~cm}^{-1}$.

When comparing these data, it is obvious that in spectrum of native starch oscillation frequency at $981.81 \mathrm{~cm}^{-1}$ has increased and in spectrum of modified starch it lies at $1024.82 \mathrm{~cm}^{-1}$; the band with oscillation frequency of 923.07 $\mathrm{cm}^{-1}$ has shifted to $866.66 \mathrm{~cm}^{-1}$ and the band with frequency of oscillations of $609.79 \mathrm{~cm}^{-1}$ has shifted to $672.22 \mathrm{~cm}^{-1}$, indicating change in noncharacteristic region of native starch sample spectrum after acylation. In IR spectrum there are no signals of acylchloride group $\left(1785-1815 \mathrm{~cm}^{-1}\right)$ and those of hydrolysis products of acetylmalic acid chloroanhydride, namely, oscillation band of carboxylic acid $\left(1750-1770 \mathrm{~cm}^{-1}\right.$ and their salts $1640 \mathrm{~cm}^{-1}$ ). This allows assuming that glucopyranose rings have been crosslinked due to reaction of both chloroanhydride groups.

Consequently, a sample of native and modified starches has a different chemical composition as a result of potato starch chemical modification by acetylmalic acid chloroanhydride. 


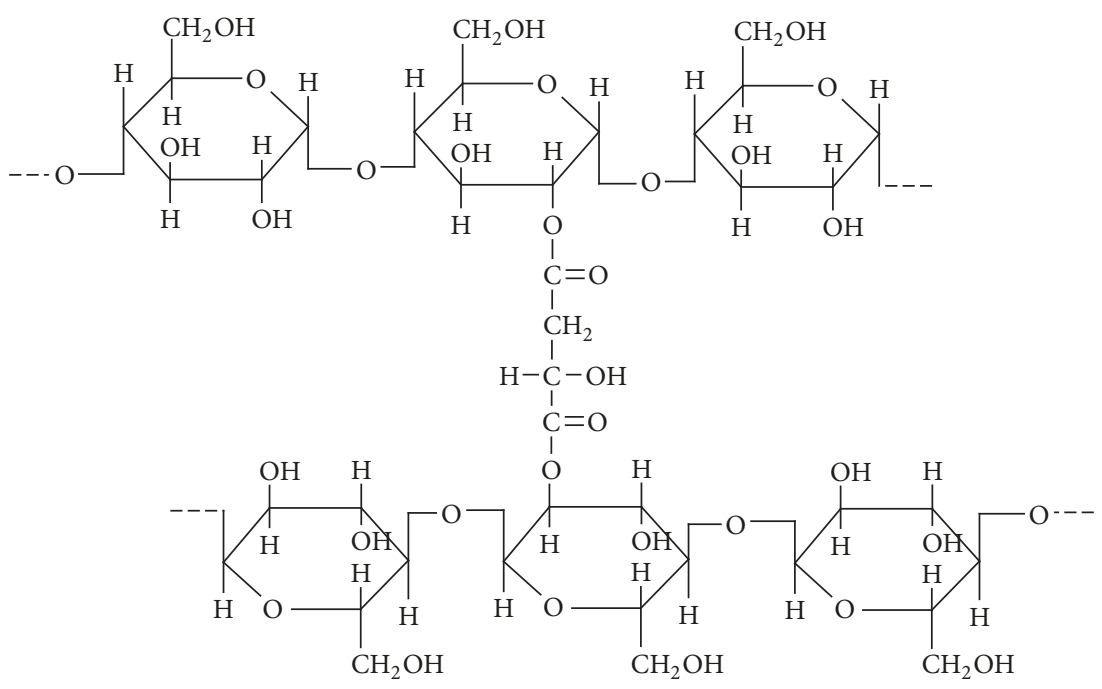

FIGURE 2: Structural formula of potato starch modification product.

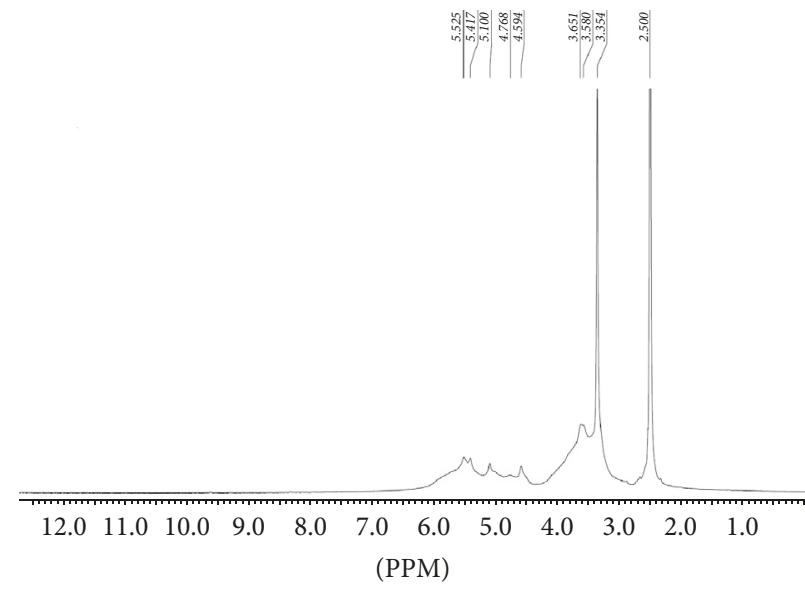

(a)

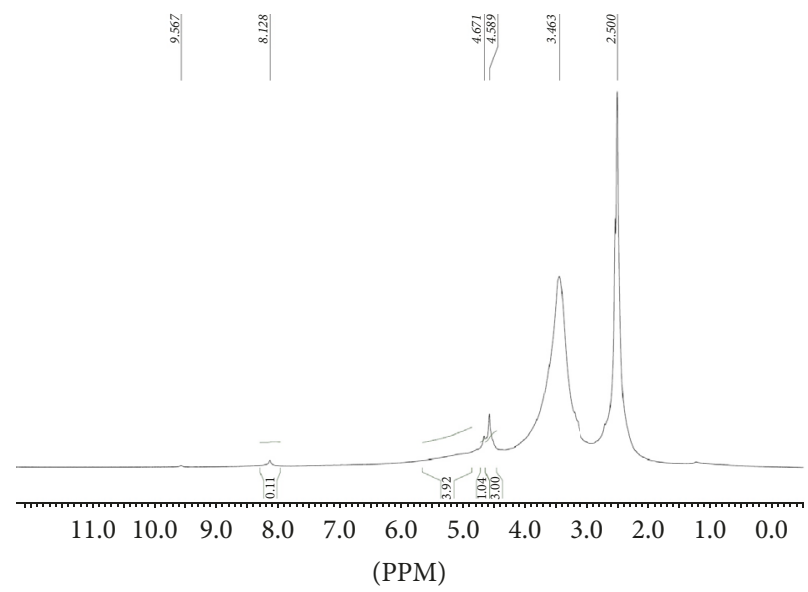

(b)

FIGURE 3: NMR spectra of native (a) and modified (b) potato starch.

4.2. Elemental Analysis. The obtained results of elemental analysis of acetylated potato starch by malic acid chloride are as follows: $44.47 \%$ of carbon has been found, $6.07 \%$ of hydrogen has been defined; $44.86 \%$ of carbon and $5.79 \%$ of hydrogen have been calculated. The results presented and infrared spectra (Figure 1) show that starch acylation by malic acid chloride was carried out by crosslinking of glucopyranose starch chains by malic acid chloride through the ratio of one molecule of malic acid chloride and six residues of glucopyranose rings (see Figure 2).

According to the previous paper [14], the most easily acetylated is a primary alcohol group and a hydroxyl alcohol group at $C_{2}$. The authors [5] prefer the hydroxyl group at $C_{2}$ to the former one.

4.3. NMR Research. On the basis of NMR spectrum of potato starch acylation product by malic acid chloride (Figure 3(b)) it is clear that there is no signal at $1 \mathrm{ppm}$ which is characteristic for group $-\mathrm{CH}_{3}$ within acetyl group.
Consequently, we can faithfully state that during acylation reaction of starch by acetylmalic acid chloroanhydride its deacetylation has occurred, which correlates perfectly with the data of elemental analysis. Determining the degree of crosslinking of glucopyranose starch chains and defining the position of the substituent on NMR spectrum have appeared to be impossible.

4.4. X-Ray Structural Study. In order to find out the influence of esterification process of malic acid chloroanhydride on potato starch structure crystallinity, X-ray diffraction analysis has been used [15]. The obtained diffractograms are shown in Figure 4.

X-ray of native starch (Figure 4) indicates its amorphouscrystalline structure with the degree of crystallinity of $12 \%$. Modified starch has an amorphous structure with the degree of crystallinity only of $4 \%$, which is quite logical, since the modification is carried out at the temperature $\left(90^{\circ} \mathrm{C}\right)$ higher than that of gelatinization $\left(59^{\circ} \mathrm{C}\right)$ of potato starch. 


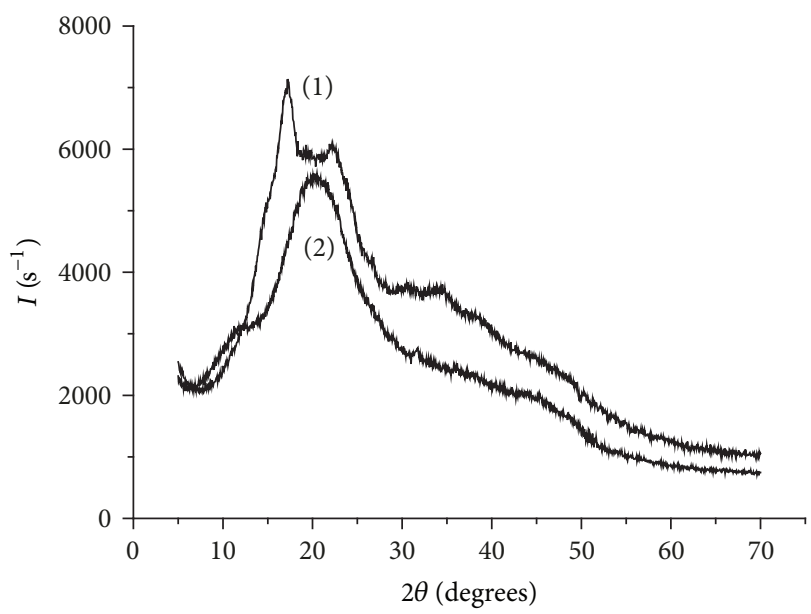

Figure 4: X-ray native (1) and modified (2) starches.

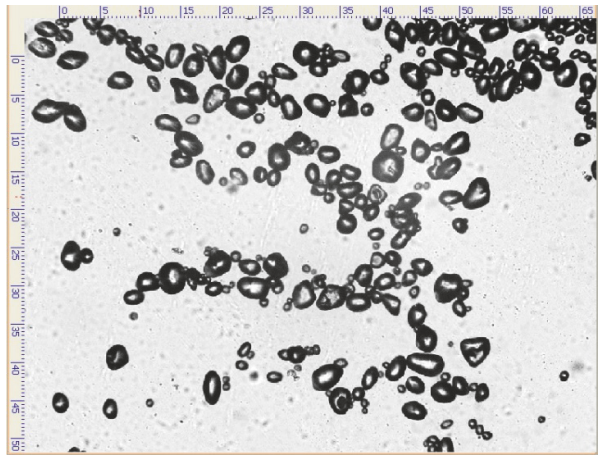

(a)

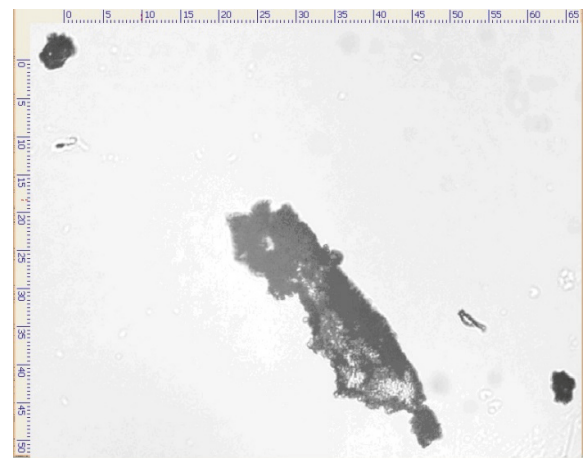

(b)

FIGURE 5: Appearance of grains of native (a) potato and modified (b) starches magnified $\times 400$.

In addition, the destruction of primary structure of starch grains is confirmed by studies using a microscope shown below. Consequently, chemical modification by acetylmalic acid results in reduction of starch crystalline structure.

4.5. Thermogravimetric Study. With the change of crystalline structure for amorphous one, we can expect a change in properties of substance to bind and retain water. Using thermal analysis some derivatograms have been obtained; the analysis of results is shown in Table 1.

According to results (Table 1), modified starch contains adsorbed water by $1.2 \%$ more due to modification in aqueous medium, and, due to this, the amount of crystallization water in modified starch is $9.7 \%$ higher. During modification, there is starch gelatinization, and when it is crosslinked, water is also retained. Due to the presence of water crystallization in modified starch, the amount of anhydrous products is lower and, as a result, in the third zone of thermolysis, the amount of anhydrous products is $11 \%$ less than that of native starch. In addition, thermolysis of anhydrous products begins at slightly lower temperature of $230^{\circ} \mathrm{C}$, as compared with $300^{\circ} \mathrm{C}$ for native starch. Consequently, modification of potato starch by acetylmalic acid leads to the change in a shape and amount of potato starch water.
4.6. Research with a Microscope. Native starch is in the form of grains that can be seen under a microscope. For each species, depending on its origin, it has a different shape and size. As in the course of modification crosslinking of glucopyranose chains has occurred, so, one can also expect changes in the shape of grains. The results of the study are shown in Figure 5.

The appearance (Figure 5) shows that the carried out potato starch chemical modification by means of crosslinking causes destruction of initial form of grains, which is also confirmed by X-ray diffraction analysis. Consequently, change in the external shape of starch grains also confirms chemical modification by acetylmalic acid.

\section{Conclusions}

Modification of potato starch by acetylmalic acid chloride has been carried out and confirmed by elemental analysis. In infrared spectra, changes in native starch frequency oscillations in noncharacteristic region have occurred: frequency of oscillations at $981.81 \mathrm{~cm}^{-1}$ has increased and in spectrum of modified starch is at $1024.82 \mathrm{~cm}^{-1}$; the band with frequency of oscillations of $923.07 \mathrm{~cm}^{-1}$ has shifted to $866.66 \mathrm{~cm}^{-1}$; and 


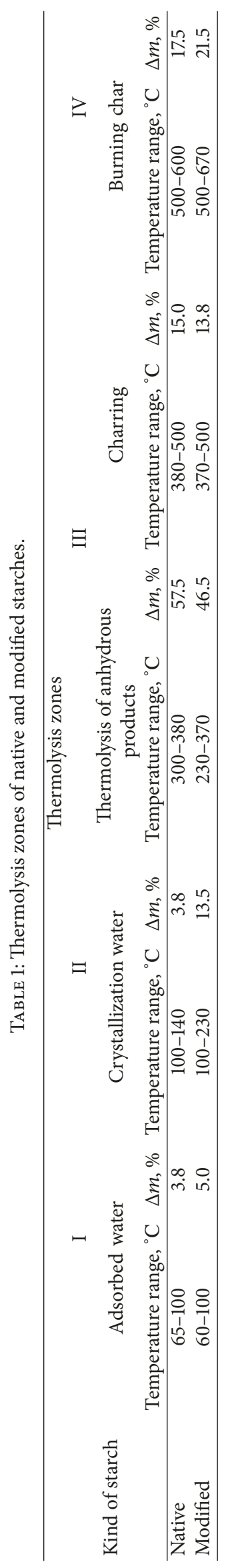


the band with that of $609.79 \mathrm{~cm}^{-1}$ has shifted to $672.22 \mathrm{~cm}^{-1}$, indicating the change in noncharacteristic region of native starch sample after acylation.

The properties of obtained modified product have been studied and it has been found that modification has changed the shape of moisture bonds with starch, the appearance of grains. The degree of crystallinity has decreased from 12 to $4 \%$.

\section{Conflicts of Interest}

The authors, Oksana Shulga, Natalya Simurova, Sergii Shulga and Jelyzaveta Smirnova, declare that there are no conflicts of interest regarding the publication of this paper.

\section{References}

[1] H. Chi, K. Xu, X. Wu et al., "Effect of acetylation on the properties of corn starch," Food Chemistry, vol. 106, no. 3, pp. 923-928, 2008.

[2] R. Santayanon and J. Wootthikanokkhan, "Modification of cassava starch by using propionic anhydride and properties of the starch-blended polyester polyurethane," Carbohydrate Polymers, vol. 51, no. 1, pp. 17-24, 2003.

[3] R. Bhosale and R. Singhal, "Process optimization for the synthesis of octenyl succinyl derivative of waxy corn and amaranth starches," Carbohydrate Polymers, vol. 66, no. 4, pp. 521-527, 2006.

[4] K. Dzulkefly, "Chemical modification of SAGO starch by solventless esterification with fatty acid chlorides," Malaysian Journal of Analytical Sciences, vol. 11, no. 2, pp. 395-399, 2007.

[5] L. Junistia, A. K. Sugih, R. Manurung, F. Picchioni, L. P. B. M. Janssen, and H. J. Heeres, "Synthesis of higher fatty acid starch esters using vinyl laurate and stearate as reactants," Starch Starke, vol. 60, no. 12, pp. 667-675, 2008.

[6] J. Aburto, I. Alric, and E. Borredon, "Preparation of Long-chain Esters of Starch Using Fatty Acid Chlorides in the Absence of an Organic Solvent," Starch - Starke, vol. 51, no. 4, pp. 10-1002, 1999.

[7] F. Fathi and H. Namazi, "Characterization and solvent freesynthesis of modified potato starch," Journal of Materials Chemistry Home, vol. 2, pp. 11-15, 2014.

[8] H. Namazi, F. Fathi, and A. Dadkhah, "Hydrophobically modified starch using long-chain fatty acids for preparation of nanosized starch particles," Scientia Iranica, vol. 18, no. 3 C, pp. 439-445, 2011.

[9] A. K. Sugih, Synthesis and Properties of Starch Based Biomaterials, University Library Groningen [Host], 2008.

[10] J. M. Fang, P. A. Fowler, J. Tomkinson, and C. A. S. Hill, "The preparation and characterisation of a series of chemically modified potato starches," Carbohydrate Polymers, vol. 47, no. 3, pp. 245-252, 2002.

[11] B. Suart, "Infrared Spectroscopy: Fundamental and Applications," 2004.

[12] R. Silversteyn, F. Vebster, and D. Kiml, "Spectrometric Identification of Organic Compounds," 2011.

[13] V. A. Mironov and S. A. Yankovskiy, "Spectroscopy in organic chemistry," Collection of Tasks, 1985.

[14] V. Myllymaki and R. Aksela, U.S. Patent Application No. 10/566.

[15] Yu. S. Lipatov, V. V. Shilov, Yu. P. Gomza, and N. E. Kruglyak, "X-ray methods for studying polymer systems," 1982. 


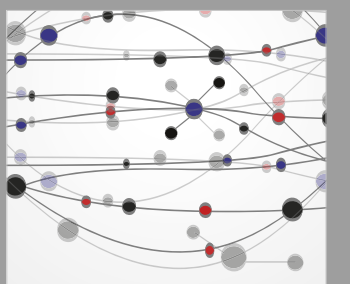

The Scientific World Journal
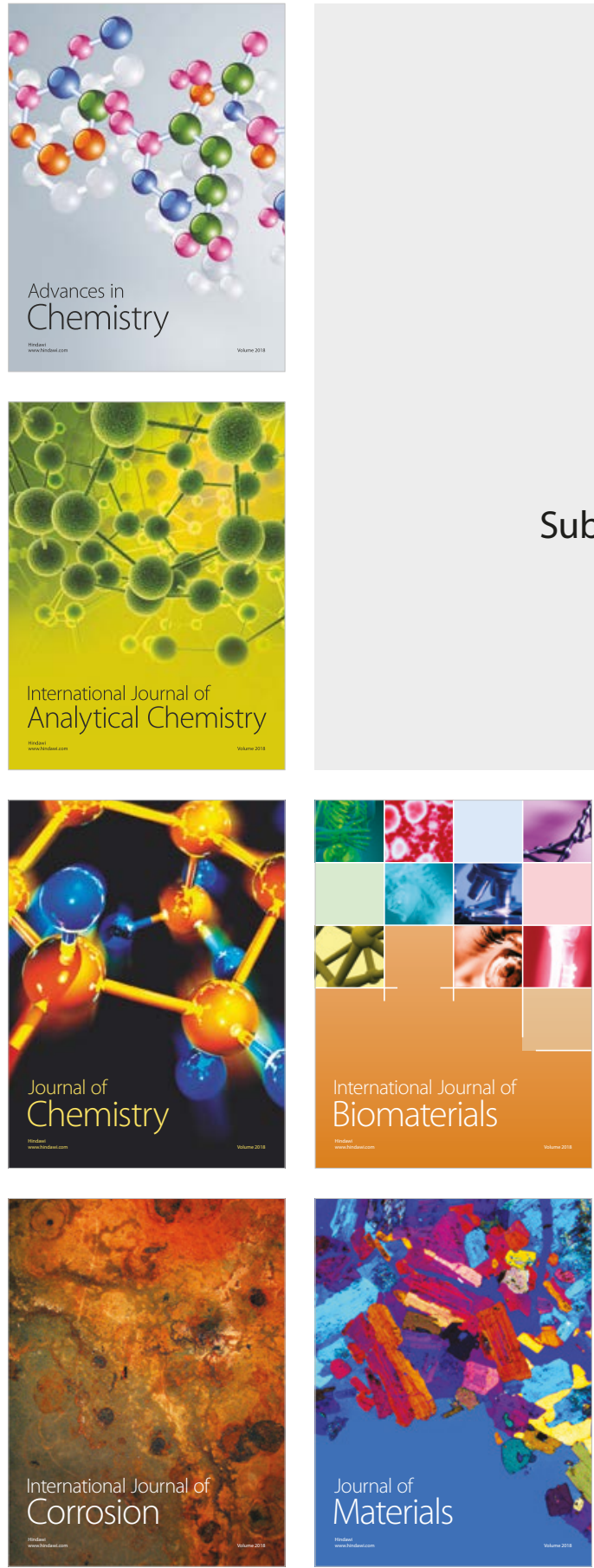

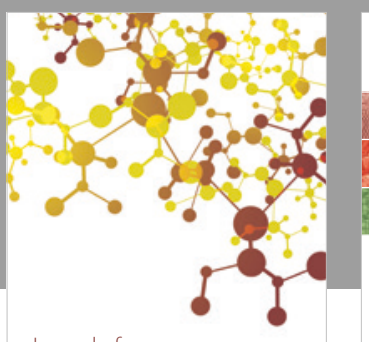

Journal of

Applied Chemistry
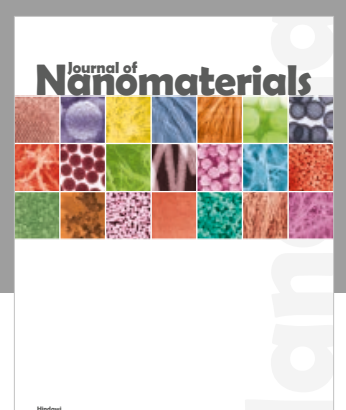

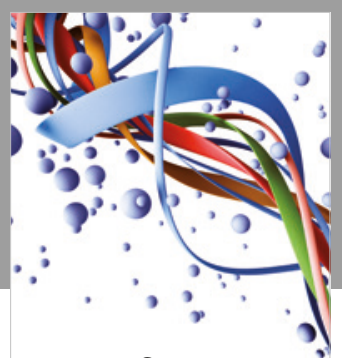

Scientifica

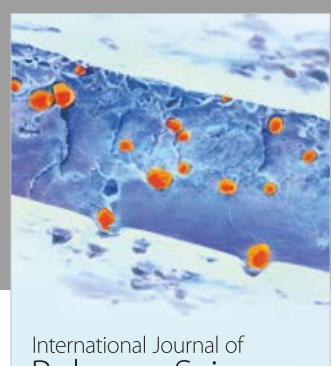

Polymer Science

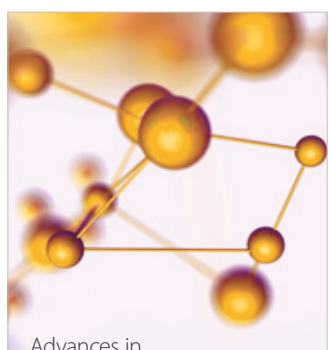

Physical Chemistry
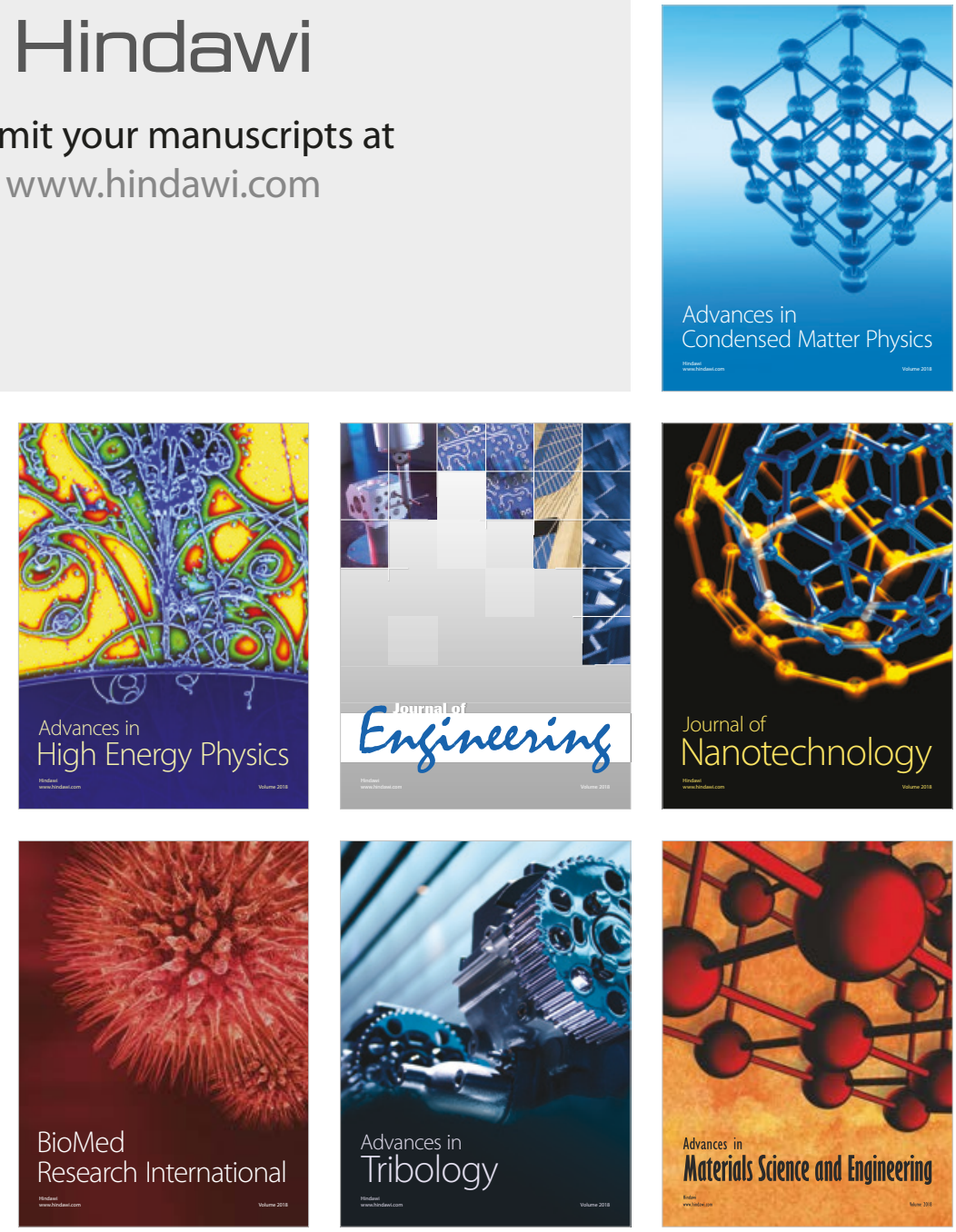